\title{
Impact Des Teneurs en Nitrates sur le Taux de Desserte en Eau Potable des Populations en Zone du Socle de Damagaram Mounio (Est du Niger)
}

\author{
Abdou Hamidou, \\ Sandao Issoufou, \\ Ousmane Boureima,
}

Université Abdou Moumouni, Faculté des sciences et techniques, département de géologie, Laboratoire des chimies des eaux, Niamey, Niger

\section{Résumé}

Le socle du Damagaram-Mounio, zone d'étude, est situé dans la partie Est de la région de Zinder. Dans cette zone, les aquifères discontinus sont localisés dans des horizons fracturés, fissurés et/ou altérés des formations granitoïdes et métamorphiques du Précambrien et des granites jeunes fissurés. Ils sont captés essentiellement par des forages dont les taux d'échec, très élevés, peuvent dépasser les 50\%. Par ailleurs, les débits de forages positifs sont inférieurs à $2 \mathrm{~m}^{3} / \mathrm{h}$ à plus de $85 \%$ d'entre eux. Ainsi, le problème de ressource en eau se pose avec acuité et le taux de couverture en eau est l'un des plus faibles du pays $(57,46 \%)$, alors que la moyenne nationale est de 70 , $85 \%$. Dans cette zone très peuplées, ce manque d'eau est aggravé par les teneurs de certains paramètres physico chimiques dont les nitrates d'où la présente étude qui a pour objectif principal la détermination des pourcentages des forages à forte teneurs en nitrates afin de connaitre leur impact sur le taux de desserte en eau potable des populations en zone du socle de Damagaram Mounio. A l'issue des mesures in situ des paramètres physiques, des analyses chimiques des échantillons des eaux de nappes, l'interprétation des donnés a permis d'établir que 32\% des points d'eau modernes échantillonnés présentent des teneurs en nitrates dépassant la limite fixée par l'OMS pour une eau de boisson $(50 \mathrm{mg} / \mathrm{l})$, par conséquent, ces eaux sont non conforme à la consommation humaine. La distribution spatiale des teneurs en nitrates dans les eaux souterraines de la zone a montré que celles-ci sont plus accentuées entre les villes de Damagaram Takaya et Birni Kazoé qui se caractérisent par des valeurs supérieures à $100 \mathrm{mg} / \mathrm{l}$. Par ailleurs, la relation entre les nitrates et les valeurs de la conductivité électrique, a montré que la minéralisation des eaux souterraines de la zone est contrôlée par les ions nitrates. Enfin, les teneurs isotopiques en ${ }^{15} \mathbf{N}$ obtenues dans les eaux de la zone d'étude varient de 
0 à 7,2, montrant ainsi que les nitrates pourraient provenir des engrains chimiques azotés et /ou de la nature du sol.

Mots clés: Aquifère discontinu, Taux de desserte, Nitrates, Eau potable, Damagaram Mounio

\title{
Impact of Nitrate Levels on the Rate of Drinking Water Supply for Populations in the Damagaram Mounio Basement Area
}

\author{
Abdou Hamidou, \\ Sandao Issoufou, \\ Ousmane Boureima,
}

Université Abdou Moumouni, Faculté des sciences et techniques, département de géologie, Laboratoire des chimies des eaux, Niamey, Niger

\begin{abstract}
The basement of the Damagaram-Mounio study area is located in the eastern part of the Zinder region. In this zone, the discontinuous aquifers are located in fractured, cracked and / or altered horizons of the Precambrian granitoids and metamorphic formations and the fissured young granites. They are mainly captured by boreholes whose failure rates, which are very high, can exceed $50 \%$. In addition, more than $85 \%$ of positive drilling flows are less than $2 \mathrm{~m} 3 / \mathrm{h}$. Thus, the problem of water resources is acute and the rate of water coverage is one of the lowest in the country $57.46 \%$, while the national average is $70.85 \%$. In this densely populated area, this lack of water is aggravated by the contents of certain physico-chemical parameters in particular the nitrates hence the present study which main objective is to determine the percentages of boreholes with high nitrate contents in order to know their impact on the rate of drinking water supply for populations in the Damagaram Mounio basement area. After in situ measurements of physical parameters, chemical analyzes of groundwater samples, interpretation of the data made it possible to establish that $32 \%$ of modern water points sampled have nitrate contents exceeding the limit set by who for drinking water $(50 \mathrm{mg} / 1)$, therefore, these waters are unsuitable for human consumption. The spatial distribution of nitrate contents in the groundwater of the area has shown that it is more accentuated between the cities of Damagaram Takaya and Birni Kazoé which are characterized by values higher than $100 \mathrm{mg} / \mathrm{l}$. Furthermore, the
\end{abstract}


relationship between nitrates and the values of electrical conductivity has shown that the mineralization of groundwater in the area is controlled by nitrate ions. Finally, the isotopic contents of $15 \mathrm{~N}$ obtained in the waters of the study area vary from 0 to 7.2 , thus showing that the nitrates could come from nitrogenous chemical substances and / or from the nature of the soil.

Keywords: Discontinuous aquifer, Service rate, Nitrates, Drinking water, Damagaram Mounio

\section{Introduction}

$\mathrm{Au}$ Sahel et en particulier au Niger, les conditions climatiques aléatoires et la forte croissance de la population augmentent la demande en eau. Dans les zones du socle du Niger, comme le Damagaram Mounio, le Sud Maradi et le Liptako Gourma, la disponibilité de l'eau, est gravement entravée par les conditions géologiques (Ousmane, 1988 ; Babayé 2013). En effet, en plus du taux élevé des forages négatifs en particulier dans le Damagaram Mounio, zone de l'étude, la qualité physico chimique des eaux des plusieurs forages est inapte à la consommation (Ousmane, 1988). Ainsi pour satisfaire leur besoin quotidien en eau d'alimentation, en eau d'irrigation et d'abreuvage des animaux, les populations sont contraintes de faire recours à des aquifères profonds situés à plusieurs dizaines de mètre ou des nappes alluviales, sources de nombreuses maladies d'origine hydrique. Il parait donc urgent d'entreprendre des investigations sur la concentration de teneurs en nitrates dans les ressources en eau souterraine de la zone. En effet, il s'agit d'une réelle préoccupation pour les populations de cette région, car le taux de couverture en eau potable est l'un de plus faibles du pays $57,46 \%$ alors que la moyenne nationale est de 70,85\%. Cette étude dont l'objectif principale est de déterminer les pourcentages des forages à forte teneurs en nitrates afin de connaitre leur impact sur le taux de desserte en eau potable des populations en zone du socle de Damagaram Mounio s'inscrit dans cette perspective. Les objectifs spécifiques visent à inventorier les points d'eaux modernes captant les aquifères discontinus $\mathrm{du}$ socle, la détermination des paramètres physicochimiques, de la relation entre les nitrates avec certains paramètres hydro chimiques et enfin de l'origine des nitrates.

\section{Matériel et Méthodes}

\section{Présentation de la zone d'étude}

Le socle du Damagaram-Mounio est situé dans la partie Est de la région de Zinder. Cette zone se situe entre $9^{\circ}$ et $9^{\circ} 35^{\prime}$ Est, $13^{\circ} 45^{\prime}$ et $14^{\circ} 10^{\prime}$ Nord, couvre une superficie d'environ $10000 \mathrm{~km}^{2}$ (Figure 1). Dans cette zone, les aquifères discontinus sont localisés dans des horizons fracturés, fissurés et/ou altérés des formations granitoïdes et métamorphiques du 
Précambrien et des granites jeunes fissurés. Ils sont captés essentiellement par des forages dont les taux d'échec, très élevés, peuvent dépasser les $50 \%$ et les débits de forages positifs sont inférieurs à $2 \mathrm{~m}^{3} / \mathrm{h}$ à plus de $85 \%$ d'entre eux (Ousmane, 1988).

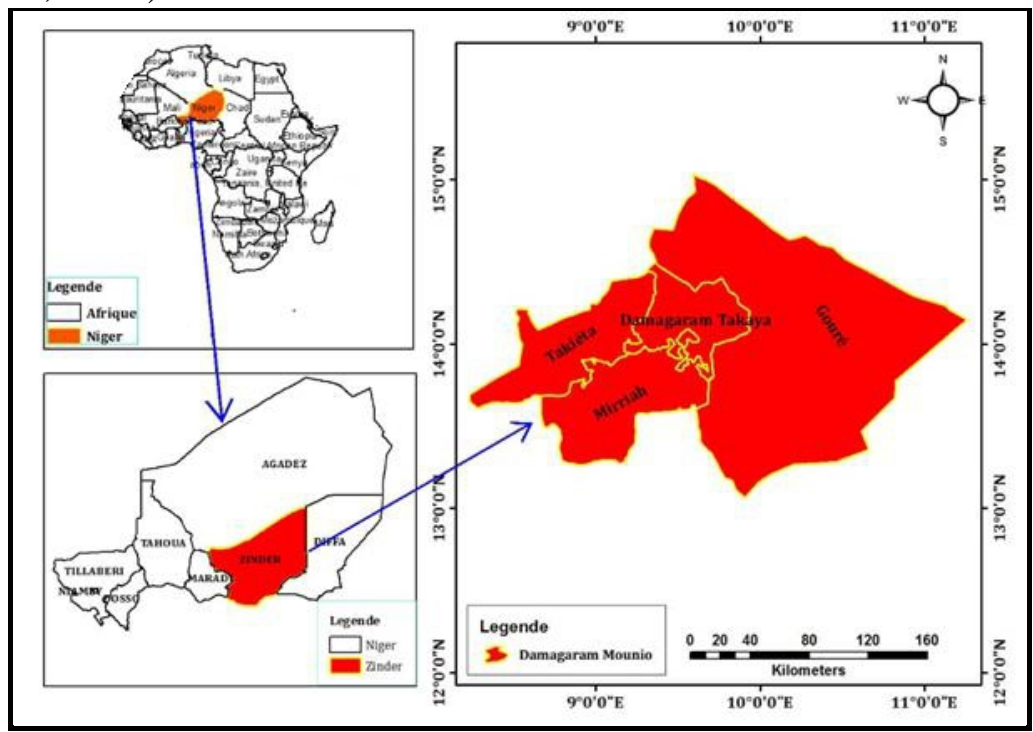

Figure 1 : Carte de localisation de la zone d'étude

La région du Damagaram Mounio, couvre trois (3) grandes provinces hydrogéologiques qui sont (Ousmane, 1988 ; BRGM, 1983 ; Kruger, 1977) : la province du Continental Intercalaire au Nord et à l'Ouest, celui du socle cristallin et cristallophyllien au centre et enfin celui du Bassin du Lac-Tchad au Sud et à l'Est (figure 2).

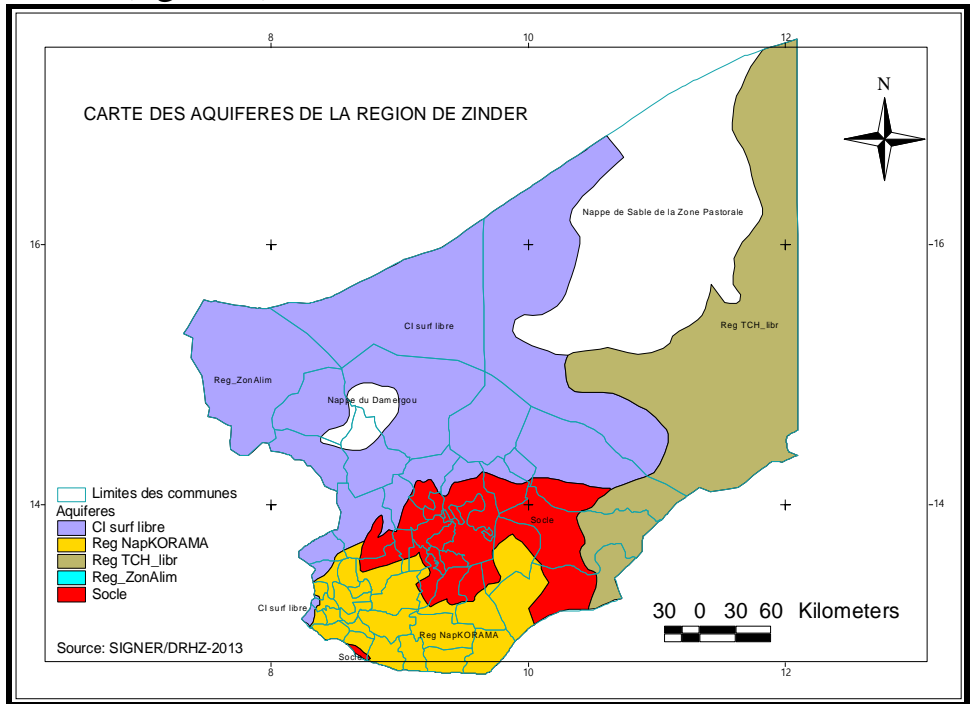

Figure 2 : Carte des systèmes aquifères de la région de Zinder (DRH, 2013) 


\section{Méthodologie}

Les données qui sont utilisées dans le cadre de cette étude sont de sources multiples ; certaines sont fournies par la Direction Régionale de l'Hydraulique (DRH) de Zinder. En effet, dans le cadre du suivi de la qualité des eaux souterraines de la région de Zinder, neuf (9) échantillons prélevés dans certains ouvrages de la zone d'étude ; ont fait l'objet d'une étude isotopique. Ces échantillons ont été analysés dans le cadre du projet national assisté par l'Agence Internationale de l'Energie Atomique (AIEA). D'autres sont issus de rapports existants. Par ailleurs, une campagne d'échantillonnage d'eau a été effectuée en mai (2017), période de basse eau et quarante-un (41) points d'eau modernes (forages équipés de Pompe à Motricité Humaine et Puits Cimentés) ont été échantillonnés dans les zones fissurées et où fracturées du socle de Damagaram Mounio. Les quarante-un (41) ouvrages sont repartis comme suit :

$\checkmark$ Quarante (40) forages nommés (F);

$\checkmark$ Un (1) Puits Cimentés nommés (PC).

Les échantillons d'eaux ont été prélevés dans deux flacons poly éthyléniques de $11 \mathrm{ml}$ préalablement stérilisés. Ensuite les paramètres $\left(\mathrm{T}^{\circ}, \mathrm{CE}, \mathrm{pH}\right)$ de ces différents échantillons ont été mesurés sur le terrain respectivement à l'aide de conductimètre, $\mathrm{pH}$-mètre (initialement calibrés pour les quatre derniers). Les échantillons d'eau prélevée ont été conservés au frais dans des glacières à la température $4^{\circ} \mathrm{C}$ et transportés au laboratoire de la Direction Régionale 1'Hydraulique (DRH) de Zinder pour analyse. Un délai de 48 heures à compter de la date d'arrivée au laboratoire des échantillons, ont été fixé pour analyse complète de tous les paramètres définis. Ainsi, les ions Calcium $\left(\mathrm{Ca}^{2+}\right)$ et Magnésium $\left(\mathrm{Mg}^{2+}\right)$ sont déterminés par titrimétrie avec les réactifs spécifiques correspondants. Pour le calcium, c'est le calver 2 qui a été utilisé comme réactif, alors que pour le magnésium, c'est le Hard 9 ; les ions Potassium $\left(\mathrm{K}^{+}\right)$et Sodium $\left(\mathrm{Na}^{+}\right)$, sont déterminées par photométrie à flamme avec un spectrophotomètre de type JENWAY PFP7 ; les ions de fer $\left(\mathrm{Fe}^{2+}\right)$, de nitrates $\left(\mathrm{NO}_{3}{ }^{-}\right)$, de sulfates $\left(\mathrm{SO}_{4}{ }^{2-}\right)$ et de chlorures $\left(\mathrm{Cl}^{-}\right)$sont déterminés par spectrophotométrie avec le spectrophotomètre DR 2800 et des réactifs respectifs qui sont : fer rover, nitraver5, sulfaver, mercuric thiocyanate+ ferrie ion solution. Toutes ces analyses ont été réalisées suivant le protocole décrit par Jean Rodier (2009). L'ensemble des données collectées sur les eaux souterraines de la zone d'étude, a permis de caractériser les teneurs en nitrates. Le traitement des données a nécessité un travail de laboratoire, avec des logiciels tels que ArcGIS pour la cartographie.

\section{Résultats et Discussion}

Les résultats analytiques de quarante-un (41) points d'eau moderne échantillonnée dans le cadre de cette étude, dans la zone du socle de Damagaram Mounio, sont présentés dans les tableaux 1 et 2 ci-dessous : 


\begin{tabular}{|c|c|c|c|c|c|c|c|c|c|c|c|c|c|c|c|}
\hline $\mathbf{N}^{\circ}$ & Villages & Libellé & $\mathbf{T}^{\circ}$ & pH & CE en $\mu / \mathrm{cm}$ & $\mathrm{Ca}^{2+}$ & $\mathrm{Mg}^{2+}$ & $\mathrm{Na}^{2+}$ & $\mathbf{K}^{+}$ & $\mathrm{Fe}^{2+}$ & $\mathrm{HCO}_{3}^{-}$ & $\mathrm{Cl}^{-}$ & $\mathrm{SO4}^{2-}$ & $\mathrm{NO}_{3}^{-}$ & Aquifères \\
\hline 1 & Afnori & Forage & 31,60 & 7,32 & 1627,00 & 157,00 & 0,90 & 297,00 & 5,40 & 0,00 & 927,00 & 15,00 & 50,00 & 49,24 & Granite \\
\hline 2 & Albarkaram & Forage & 28,60 & 6,30 & 1827,00 & 157,00 & 0,90 & 8,00 & 5,40 & 0,30 & 927,00 & 12,00 & 35,00 & 268,40 & Granite \\
\hline 3 & Atchafa & Forage & 31,00 & 7,10 & 504,00 & 154,00 & 0,90 & 101,00 & 18,00 & 0,00 & 196,00 & 15,00 & 50,00 & 34,32 & Granite \\
\hline 4 & Boubout & Forage & 31,50 & 6,70 & 628,00 & 195,00 & 39,00 & 202,00 & 18,60 & 0,00 & 192,00 & 15,00 & 50,00 & 101,20 & Granite \\
\hline 5 & Bougoum & Forage & 30,80 & 7,20 & 1595,00 & 134,00 & 20,00 & 202,00 & 14,50 & 0,00 & 192,00 & 16,00 & 50,00 & 88,40 & Granite \\
\hline 6 & Bourbourwa & Forage & 32,10 & 5,60 & 2680,00 & 34,20 & 20,00 & 20,00 & 14,50 & 0,30 & 192,00 & 30,00 & 50,00 & 660,00 & Granite \\
\hline 7 & Chirkou Garin & Forage & 31,10 & 7,23 & 940,00 & 20,50 & 20,00 & 15,00 & 14,50 & 0,00 & 192,00 & 23,00 & 50,00 & 47,00 & Granite \\
\hline 8 & Dan kourtchi & Forage & 32,70 & 6,47 & 284,00 & 20,50 & 20,00 & 84,60 & 4,10 & 0,00 & 472,00 & 28,00 & 50,00 & 0,35 & Granite \\
\hline 9 & Dankeni Gambo & Forage & 31,10 & 7,01 & 875,00 & 212,00 & 20,00 & 75,00 & 4,10 & 0,00 & 472,00 & 111,00 & 55,00 & 68,20 & Gneiss \\
\hline 10 & Danladi & Forage & 31,60 & 6,24 & 1751,00 & 123,10 & 21,10 & 20,00 & 8,00 & 0,20 & 184,68 & 30,00 & 9,00 & 204,60 & Granite \\
\hline 11 & Daratcham & Forage & 32,00 & 6,70 & 353,00 & 61,50 & 5,00 & 126,00 & 3,10 & 0,00 & 10,80 & 150,00 & 200,00 & 37,84 & Granite \\
\hline 12 & Djeouta Bougagé & Forage & 28,50 & 7,21 & 203,00 & 456,80 & 150,50 & 126,00 & 62,50 & 1,00 & 423,00 & 900,00 & 1100,00 & 12,32 & Granite \\
\hline 13 & Doufoufouk & Forage & 27,80 & 6,54 & 703,00 & 230,00 & 381,00 & 126,00 & 65,00 & 1,20 & 168,00 & 593,00 & 2800,00 & 42,67 & Granite \\
\hline 14 & Dungass & Forage & 30,90 & 7,30 & 237,00 & 230,00 & 381,00 & 126,00 & 3,40 & 0,00 & 8,20 & 157,00 & 180,00 & 3,96 & Granite \\
\hline 15 & Dogo Maikassoua & Forage. & 28,90 & 7,28 & 249,00 & 230,00 & 381,00 & 55,00 & 5,70 & 0,00 & 217,00 & 30,00 & 61,00 & 27,00 & Granite \\
\hline 16 & Faya Haoussa & Forage & 32,20 & 7,80 & 1131,00 & 230,00 & 381,00 & 55,00 & 3,20 & 0,00 & 217,00 & 15,00 & 50,00 & 48,80 & Granite \\
\hline 17 & Foulatari & Forage & 31,10 & 7,10 & 234,00 & 59,20 & 3,00 & 55,00 & 4,80 & 0,03 & 83,00 & 14,00 & 24,00 & 40,80 & Granite \\
\hline 18 & Garin Malam & Forage & 30,20 & 6,50 & 727,00 & 32,80 & 18,50 & 116,00 & 5,00 & 0,01 & 381,00 & 6,00 & 20,00 & 76,40 & Granite \\
\hline 19 & Gorgoré & Forage & 31,40 & 6,96 & 575,00 & 88,90 & 2,40 & 25,00 & 5,80 & 0,20 & 71,82 & 38,00 & 50,00 & 42,80 & Granite \\
\hline 20 & Ididigouri & Forage & 28,60 & 6,30 & 1122,00 & 75,20 & 2,40 & 20,00 & 5,80 & 0,00 & 71,82 & 30,00 & 50,00 & 170,72 & Granite \\
\hline
\end{tabular}

Tableau 1 : Teneurs en nitrates des eaux de forages échantillonnés, dans le cadre de cette étude, dans la zone du socle de Damagaram-Mounio (Analyse effectuée par le laboratoire de chimie des eaux de l'Hydraulique de Zinder) 


\begin{tabular}{|c|c|c|c|c|c|c|c|c|c|c|c|c|c|c|c|}
\hline $\mathrm{N}^{\circ}$ & Noms & Libellé & $T^{\circ}$ & $\mathrm{pH}$ & CE en $\mu / \mathrm{cm}$ & $\mathrm{Ca}^{2+}$ & $\mathrm{Mg}^{2+}$ & $\mathrm{Na}^{2+}$ & $\mathrm{K}^{+}$ & $\mathrm{Fe}^{2+}$ & $\mathrm{HCO}_{3}^{-}$ & $\mathrm{Cl}^{-}$ & $\mathrm{SO}_{4}^{2-}$ & $\mathrm{NO}_{3}^{-}$ & Aquifères \\
\hline 21 & Kakissara & Forage & 32,70 & 6,90 & 909,00 & 95,80 & 2,40 & 15,00 & 5,80 & 0,10 & 71,82 & 23,00 & 50,00 & 49,72 & Granite \\
\hline 22 & Kalimbodi & Forage & 33,40 & 7,80 & 231,00 & 48,40 & 66,80 & 62,30 & 5,60 & 0,09 & 335,00 & 61,00 & 22,00 & 16,72 & Granite \\
\hline 23 & Kasoé & Forage & 31,20 & 5,60 & 1116,00 & 48,40 & 66,80 & 62,30 & 6,90 & 0,00 & 323,00 & 30,00 & 50,00 & 479,60 & Granite \\
\hline 24 & Kassama & Forage & 30,60 & 6,23 & 1368,00 & 75,20 & 66,80 & 35,00 & 6,90 & 0,00 & 323,00 & 53,00 & 27,00 & 180,4 & Granite \\
\hline 25 & Kourni & Forage & 32,00 & 7,01 & 234,00 & 0,80 & 2,00 & 171,00 & 3,50 & 0,01 & 346,00 & 2,00 & 27,00 & 0,22 & Granite \\
\hline 26 & Kourssadi & Forage & 30,10 & 6,21 & 630,00 & 61,60 & 2,00 & 15,00 & 3,50 & 0,30 & 346,00 & 23,00 & 19,00 & 43,60 & Granite \\
\hline 27 & Koussotori & Forage & 31,60 & 7,32 & 430,00 & 3,20 & 15,50 & 15,00 & 5,80 & 0,80 & 38,00 & 6,00 & 19,00 & 30,11 & Granite \\
\hline 28 & Maya Tchasko & Forage & 30,30 & 6,50 & 1250,00 & 4,00 & 12,10 & 175,00 & 2,20 & 0,20 & 410,00 & 1,00 & 28,00 & 91,20 & Granite \\
\hline 29 & Midik & Forage & 29,10 & 6,90 & 551,00 & 48,40 & 16,50 & 171,00 & 4,50 & 0,01 & 9,00 & 43,00 & 55,00 & 42,80 & Gneiss \\
\hline 30 & Naoumé & Forage & 32,20 & 6,40 & 428,00 & 38,00 & 390,00 & 153,00 & 4,90 & 0,01 & 371,00 & 5,00 & 26,00 & 124,00 & Granite \\
\hline 31 & Nassalé & Forage & 31,10 & 6,80 & 1116,00 & 88,90 & 390,00 & 35,00 & 4,90 & 0,50 & 371,00 & 53,00 & 26,00 & 123,20 & Granite \\
\hline 32 & Lassoury & Forage & 30,20 & 7,00 & 758,00 & 123,10 & 390,00 & 25,00 & 4,90 & 0,50 & 184,68 & 38,00 & 26,00 & 42,24 & Granite \\
\hline 33 & Ourak & Forage & 31,70 & 6,90 & 730,00 & 75,20 & 390,00 & 30,00 & 4,90 & 0,50 & 249,00 & 45,00 & 26,00 & 40,48 & Granite \\
\hline 34 & Sabon Roua & Forage & 28,50 & 6,27 & 941,00 & 171,00 & 34,00 & 55,00 & 3,10 & 0,00 & 2,80 & 83,00 & 51,00 & 41,12 & Granite \\
\hline 35 & Tchidassawra & Forage & 31,10 & 6,80 & 763,00 & 355,70 & 34,00 & 65,00 & 3,10 & 0,15 & 2,80 & 98,00 & 87,00 & 40,60 & Granite \\
\hline 36 & Tchiroum 1 & Forage & 25,80 & 6,80 & 690,00 & 61,60 & 10,20 & 20,00 & 3,10 & 0,20 & 454,00 & 30,00 & 0,00 & 43,71 & Granite \\
\hline 37 & Tchiroum 2 & Forage & 30,80 & 6,90 & 564,00 & 75,20 & 10,20 & 35,00 & 3,10 & 0,00 & 112,86 & 53,00 & 0,00 & 42,64 & Granite \\
\hline 38 & Tirmini & Forage & 23,30 & 6,20 & 637,00 & 328,30 & 10,20 & 100,00 & 3,10 & 0,20 & 492,50 & 150,00 & 0,00 & 6,60 & Granite \\
\hline 39 & Tirmou & Forage & 28,10 & 7,30 & 789,00 & 95,80 & 10,20 & 35,00 & 3,10 & 1,20 & 143,64 & 53,00 & 0,00 & 12,56 & Granite \\
\hline 40 & Tis & Forage & 29,70 & 7,36 & 766,00 & 95,80 & 10,20 & 29,40 & 3,90 & 0,00 & 99,00 & 15,00 & 50,00 & 27,71 & Quartzite \\
\hline 41 & Toumnia & PC & 26,30 & 7,70 & 202,00 & 97,80 & 48,40 & 29,40 & 2,80 & 0,50 & 4,40 & 15,00 & 180,00 & 37,84 & Granite \\
\hline
\end{tabular}

Tableau 2: Teneurs en nitrates des eaux de forages échantillonnés, dans le cadre de cette étude, dans la zone du socle de Damagaram-Mounio (Analyse effectuée par le laboratoire de chimie des eaux de l'Hydraulique de Zinder) 
Les résultats analytiques de quarante-un (41) points d'eau moderne échantillonnés (tableaux 1 et 2) montrent que (Figure 3) :

- Vingt-huit (28) échantillons soit $\mathbf{6 8 \%}$ des ouvrages, ont des teneurs en nitrates inferieures à la valeur limite admissible pour les eaux de consommation fixés à $50 \mathrm{mg} / \mathrm{L}$ selon les normes de l'organisation mondiale de la santé (OMS) ; ces eaux sont donc de bonne qualité sur le plan nitraté ;

- Treize (13) échantillons soit $\mathbf{3 2 \%}$ des ouvrages, ont des teneurs en nitrates supérieures à la valeur limite admissible pour les eaux de consommation fixés à $50 \mathrm{mg} / \mathrm{L}$, selon les normes de l'organisation mondiale de la santé (OMS) ; il s'agit des eaux des forages suivants : $\mathrm{F}_{2}, \mathrm{~F}_{4}, \mathrm{~F}_{5} ; \mathrm{F}_{6}, \mathrm{~F}_{9}, \mathrm{~F}_{10}, \mathrm{~F}_{18}, \mathrm{~F}_{20}, \mathrm{~F}_{23}, \mathrm{~F}_{24}, \mathrm{~F}_{28}, \mathrm{~F}_{30}$ et $\mathrm{F}_{31}$; ces eaux sont non conforme à la consommation humaine. Ainsi :

$\checkmark \quad$ sur les treize (13) échantillons, huit (8) soit $\mathbf{2 0 \%}$ des ouvrages ont de teneurs en nitrates qui varient de 100 à $660 \mathrm{mg} / \mathrm{L}$; il s'agit des eaux de forages suivants : $F_{2}, F_{4}, F_{6}, F_{10}, F_{20}, F_{23} . F_{24}$, $\mathrm{F}_{28}$ et $\mathrm{F}_{31}$; ces eaux sont de qualité mauvaise à médiocre sur le plan nitraté ;

$\checkmark$ sur les huit (8) échantillons, cinq (5) soit $12 \%$ des ouvrages ont de teneurs en nitrates qui varient de 50 à $100 \mathrm{mg} / \mathrm{L}$; ces eaux sont de qualité mauvaise sur le plan nitraté.

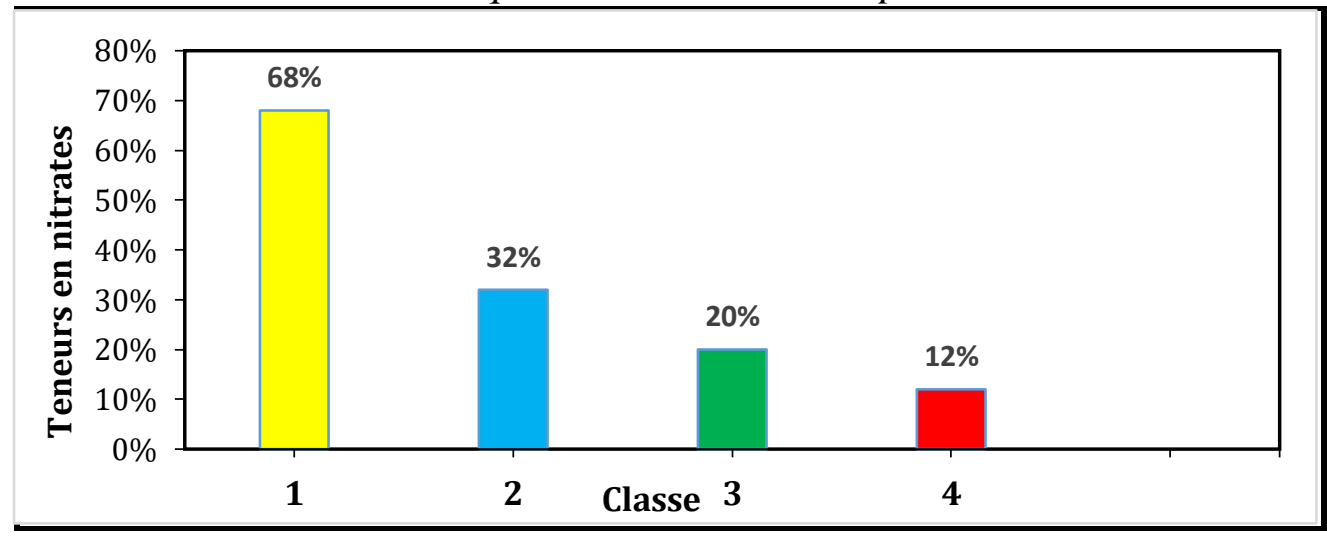

Figure 3: Classification des eaux souterraines de la zone d'étude

\section{Identification de l'origine des nitrates dans les eaux souterraines de la zone}

Les résultats isotopiques de l'Azote15 $\left({ }^{\mathbf{1 5}} \mathbf{N}\right)$ de certains points d'eau de la zone d'étude, analysés dans le cadre du projet national assisté par l'Agence Internationale de l'Energie Atomique (AIEA), sont présentés dans le tableau 3 ci-après (DRH, 2016) : 
Tableau 3: valeurs de l'Azote 15 des points d'eau de la zone d'étude

\begin{tabular}{|c|c|c|c|c|c|c|c|c|}
\hline Village & LONG & LAT & ALT & AQUIFERE & $\mathrm{NO}_{3}^{-}$ & $\begin{array}{l}\mathrm{NO}_{3}^{-} \\
\text {meq }\end{array}$ & ${ }^{15} \mathrm{~N}$ & $\begin{array}{c}\mathrm{NO}_{3}{ }^{-} \\
\mathrm{T}\end{array}$ \\
\hline Dalari & 9,3530100000000 & 14,1039200000000 & 430,00 & SOCLE & 149,16 & 2,41 & 0,00 & 1,14 \\
\hline Kassama F1 & 9,2790900000000 & 13,9891700000000 & 437,00 & SOCLE & 281,56 & 4,54 & 0,00 & $<5$ \\
\hline D. Takaya & 9,4812200000000 & 14,1346000000000 & 407,00 & SOCLE & 61,16 & 0,99 & 0,00 & 0,2 \\
\hline Koursadi & 9,6193000000000 & 14,4225670000000 & 454,00 & SOCLE & 97,43 & 1,57 & 0,00 & 0,31 \\
\hline Dan ladi & 9,0179300000000 & 13,8930200000000 & 447,00 & SOCLE & 982,80 & 15,85 & 3,20 & 6,6 \\
\hline Dogon Shuri & 9,0520400000000 & 13,9234100000000 & 455,00 & SOCLE & 69,68 & 1,12 & 3,50 & 6,8 \\
\hline Bourbourwa & 9,1380200000000 & 13,9714000000000 & 479,00 & SOCLE & 693 & 11,18 & 5,90 & 0,4 \\
\hline Albarkaram & 9,2382400000000 & 13,9735700000000 & 437,00 & SOCLE & 261,36 & 4,22 & 7,20 & $<5$ \\
\hline Birni Kazoé & 9,9694400000000 & 14,2187400000000 & 394,00 & SOCLE & 308,88 & 4,98 & 7,20 & 1,3 \\
\hline
\end{tabular}

Selon une étude similaire de la qualité des eaux de la Communauté Urbaine de Niamey (Pierre Giraud, 1996), les compositions isotopiques de ${ }^{\mathbf{1 5}} \mathbf{N}$ des eaux issues des latrines varient de $+14,7$ à $16 \%$ avec de faibles concentrations en azote totale tandis que les signatures des bourbiers autour des points d'eau sont très faibles avec des concentrations élevées en azote totale. En outre selon l'étude, les signatures isotopiques de ${ }^{15} \mathbf{N}$ des engrais chimiques et fumiers utilisés dans les zones rizicoles varient de $+0,5$ à $+3 \%$ avec des valeurs élevées en nitrates. En comparant la gamme de valeurs de ${ }^{15} \mathbf{N}$ $(+0$ à $+7,20)$ de la zone d'étude, il est à exclure l'hypothèse des latrines comme source de contamination des eaux dans la zone d'étude. Suivant ces valeurs isotopiques de ${ }^{\mathbf{1 5}} \mathbf{N}$ obtenues dans la zone d'études, l'origine des nitrates pourrait provenir de la nature du sol (bourbier autour des points d'eau et éventuellement les fourmis dans les colonnes des pompes) et engrais chimiques (urées et fertilisants utilisées dans la zone d'étude).

\section{Identification des zones à teneurs élevées en nitrates}

La distribution spatiale des teneurs en nitrates dans les eaux souterraines de la zone montre que celles-ci sont plus accentuées entre les villes de Damagaram Takaya et Birni Kazoé. La carte ci-dessous (Figure 4) met en évidence des zones à fortes teneurs en nitrates qui se caractérisent par de valeurs supérieures à $100 \mathrm{mg} / \mathrm{l}$; cela pourrait s'expliquer par l'importance de la vitesse d'infiltration à travers les fissures et les fractures du socle cristallin et cristallophyllien de la zone. 


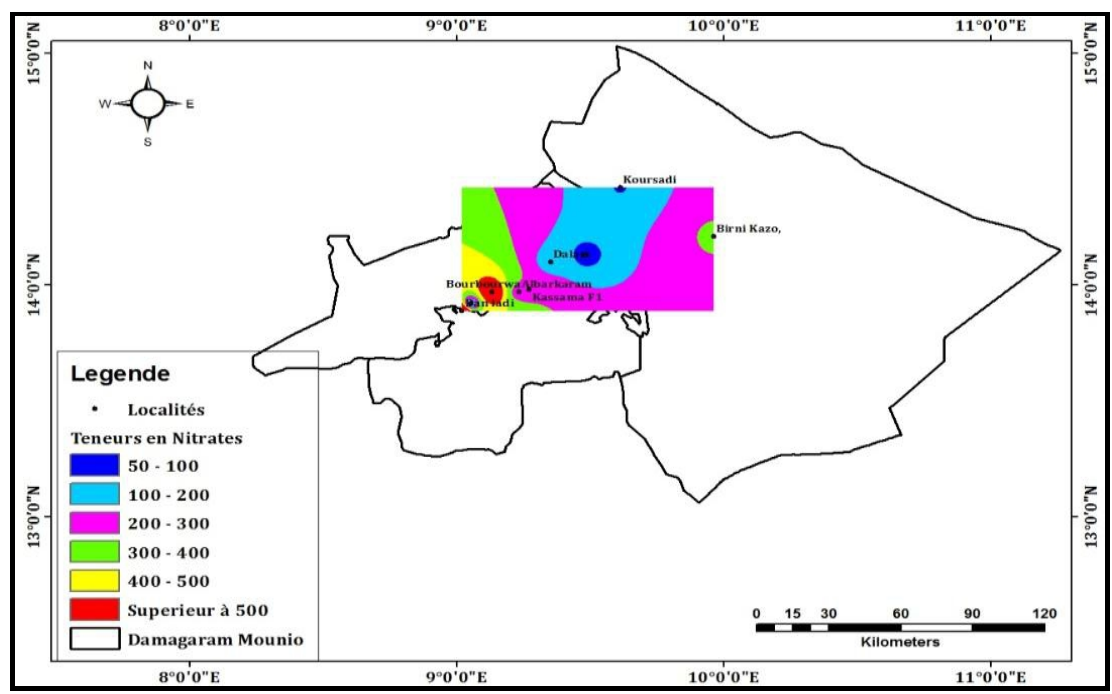

Figure 4: Carte de localisation des zones des teneurs élevées en nitrates de la zone d'étude

Rélation entre les nitrates et la minéralisation des eaux des nappes

La relation entre les nitrates et les valeurs de la conductivité électrique (figure 5), montre que la minéralisation des eaux souterraines de la zone est contrôlée par les ions nitrates, Il se dégage une relation entre les nitrates et la minéralisation des eaux des nappes et s'exprime par la relation suivante :

$$
\mathrm{NO}_{3}=0.0945 * \mathrm{CE}(\mathrm{us} / \mathrm{cm})+10.55 \quad\left(\mathrm{R}^{2}=0,74\right)
$$

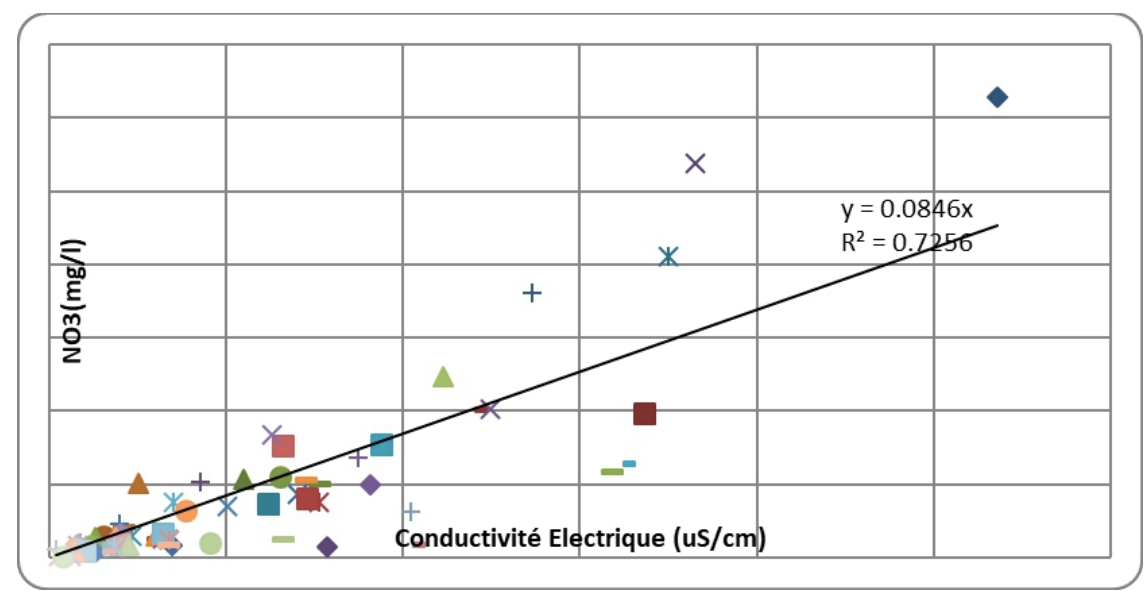

Figure 5: Rélation entre les Nitrates et la Minéralisation des eaux des nappes de la zone d'etude (DRH,2016)

\section{Conclusion}

Les résultats analytiques sur les teneurs en nitrates ont permis de mettre en évidence que $\mathbf{3 2} \%$ des ouvrages modernes échantillonnés ont des teneurs en nitrates supérieures à la valeur limite admissible pour les eaux de consommation fixés à $50 \mathrm{mg} / \mathrm{L}$, selon les normes de l'organisation mondiale 
de la santé (OMS) ; ces eaux sont de qualité mauvaise à médiocre sur le plan nitraté et sont en conséquences non conformes à la consommation humaine. La distribution spatiale des teneurs en nitrates dans les eaux souterraines de la zone, a montré que celles-ci sont plus accentuées entre les villes de Damagaram Takaya et Birni Kazoé qui se caractérisent par des valeurs supérieures à $100 \mathrm{mg} / \mathrm{l}$. Par ailleurs, la relation entre les nitrates et les valeurs de la conductivité électrique, a montré que la minéralisation des eaux souterraines de la zone est contrôlée par les ions nitrates et enfin, les teneurs isotopiques en ${ }^{15} \mathbf{N}$ obtenues dans les eaux souterraines de la zone ; varient de 0 à 7,2, montrant ainsi que les nitrates pourraient provenir des engrains chimiques azotés et /ou de la nature du sol.

\section{References:}

1. Abdou Babayé, M.S., Sandao, I., Saley, M.B., Wagani, I. \& Ousmane, B. (2016) : Comportement hydrogéochimique et contamination des eaux des aquifères fissurés du socle précambrien en milieu semi-aride (Sud-Ouest du Niger). Int. J. Biol. Chem. Sci. 10(6) : 2728-2743.

2. AHOUSSI, E.K., SORO, N., KOFFI, B.Y, SORO, G. \& BIEMI, J. (2010) : Origine de la minéralisation des eaux des aquifères discontinus sous couvert forestier de la zone Sud de la Côte d'Ivoire : cas de la région d'Abidjan-Agboville. Int. J. Biol. Chem. Sci, 4 (3) : 782-797.

3. Boukari, M. (1998) : Fonctionnement du système aquifère exploité pour l'approvisionnement en eau de la ville de Cotonou sur le littoral béninois. Impact du développement urbain sur la qualité des ressources. Thèse de doctorat, Université Cheikh Anta Diop de Dakar. Sénégal. 345p.

4. BRGM, (1983) : Projet 1000 forages, Analyse des résultats des forages par traitement statistique.

5. BRGM, (1983) : Programme hydraulique villageoise du conseil de l'entente, $1^{\text {irer }}$ phase.

6. Cadilhac, O. (1990-1991) : Atlas des ressources hydrauliques du département de Zinder, Arrondissement de Mirriah, Magaria et Matamèye. Rapport. Direction départementale de l'hydraulique de Zinder.

7. Chemseddine, F., Abderrahmane, B., Abdelkader, R. \& Elias, S. (2009) : Caractérisation hydrogéochimique des eaux souterraines du complexe aquifère Morsott-Laouinet (Région Nord de Tébessa, SudEst algérien. Afrique Science, 05 (2) 217-231.

8. Issa, S., Mama, D., Zanguina, A., Natatou, I., Boukari, M. \& Sohounhloué, D. (2015) : Hydrogeochemical characterization of Continental Intercalary Terminal, the base and the Korama aquifers 
groundwater found in five local Governments in the Zinder region in the Republic of Niger. Research Journal of Chemical and Environmental Sciences. Vol. 3, $\mathrm{n}^{\circ}$ 4. 09-16.

9. Kruger, I. (1977): Etude hydrogéologique de Damagaram Mounio et du Sud Maradi.

10. Jessen P. (1992). Analyse des conditions hydrogéologiques en vue d'un programme d'hydraulique rurale dans le département de Zinder. Rapport. Direction départementale d'hydraulique de Zinder. 344

11. Kouassi, M.A., Yao, A.K., Ahoussi, E.K., Seki, C.L., Yao, A.N., Kouassi, I.K. \& Biemi, J. (2010) : Apport des méthodes statistiques et hydrochimiques à la caractérisation des eaux des aquifères fissurés de la région du N'zi-Comoé (centre-Est de la Côte d'Ivoire). Int. J. Biol. Chem. Sci. 4 (5) : 1816-1838.

12. Ousmane, B. (1988) : Etude géochimique et isotopique des aquifères du socle de la bande sahélienne du Niger (Liptako, Sud Maradi, Zinder Est), Thèse de Doctorat d'Etat, ès Sciences Naturelles, Université de Niamey, 1988, 175 p. et annexes.

13. Pontié, M., Rumeau, M., Ndiaye, M. \& Diop, C.M. (1996) : Sur le problème de la fluorose au Sénégal : bilan des connaissances et présentation d'une nouvelle méthode de défluoruration des eaux de boisson. Cahier Santé, vo1.6: p. 27-36.

14. Pierre, G. (1996) : Etude de la qualité des eaux de la communauté urbaine de Niamey.

15. M.O. Elimination des nitrates des eaux potables, Document Technique FNDAE Hors-Série $\mathrm{N}^{\circ} 4$. Direction de l'Espace Rural et de la Forêt.

16. Rodier, J., Legube, B. \& Merlet, N. (2009) : L'analyse De L'eau (9 E Edition). Ed Dunod. Paris. 1579 pp.

17. Sanoussi, R. (2009) : Etude complémentaire en vue de l'évaluation des eaux souterraines de la région de Zinder (Niger), Rapport. Direction Régionale de l'Hydraulique de Zinder 